\title{
The $\mathrm{F}$ wave disappears due to impaired excitability of motor neurons or proximal axons in inflammatory demyelinating neuropathies
}

\author{
T Yokota, A Inaba, N Yuki, T Ichikawa, H Tanaka, Y Saito, T Kanouchi
}

\begin{abstract}
Objectives-Investigation of pathophysiology of $F$ wave disappearance in demyelinating neuropathies.

Methods-The peripheral motor nerve conduction was studied by motor evoked potential (MEP) on transcranial magnetic stimulation as well as conventional nerve conduction studies before and after the treatment in 26 patients with inflammatory demyelinating neuropathies. In addition, serum antiganglioside antibodies in the acute or active stage were examined.

Results-The F wave was abolished in 10 patients. Seven of the 10 patients showed motor evoked potentials (MEPs) on transcranial magnetic stimulation that ranged from 1-4 mV. In six of them the $F$ wave reappeared in the recovery stage, but the MEP size did not change. This may be caused by humoral factors, because the $F$ wave reappeared immediately after plasma exchange or intravenous immunoglobulin treatment. A correlation of $F$ wave disappearance with the presence of serum antiganglioside antibodies was found.

Conclusion-The major pathophysiology of $F$ wave disappearance in demyelinating neuropathies is impairment of motor neuron excitability or prolonged refractoriness of the most proximal axon for backfiring. The conventional interpretation that absent $F$ waves suggest a conduction block at the proximal site is often inadequate.
\end{abstract}

\section{(F Neurol Neurosurg Psychiatry 1996;60:650-654)}

Keywords: F wave; motor neuron excitability; GuillainBarré syndrome; motor evoked potential

It is known that the $F$ wave is typically absent during the acute stage of Guillain-Barré syndrome. ${ }^{1}$ According to the criteria of the ad hoc subcommittee of the American Academy of Neurology AIDS Task Force, ${ }^{2}$ an absent $F$ wave is one of the mandatory electrophysiological features for the diagnosis of demyelinating neuropathy. This is based on the interpretation that the disappearance of the $F$ wave indicates conduction block in the proximal nerve segments. The $F$ wave size and frequency, however, also decrease in acute cerebrovascular lesion, ${ }^{3}$ in cataplexy, ${ }^{4}$ and after chronic cerebellar stimulation, ${ }^{35}$ indicating that $F$ waves are also abolished by decreased motor neuron excitability. To clarify the pathophysiology of $F$ wave disappearance in the inflammatory demyelinating neuropathies, we used motor cortical stimulation to study motor nerve conduction through all the peripheral nerve segments. We also investigated whether the disappearance of the $F$ wave and the presence of antiganglioside antibodies were correlated.

\section{Patients and methods}

PATIENTS

Thirteen patients with Guillain-Barré syndrome, ${ }^{6} 11$ with chronic inflammatory demyelinating polyradiculoneuropathy (CIDP), ${ }^{2}$ and two with multifocal motor neuropathy $(\mathrm{MMN}),{ }^{7}$ were studied in the progressive active stage in the first one to three weeks and after treatment by plasma exchange or with intravenous immunoglobulin (IVI g; $0.4 \mathrm{~g} / \mathrm{kg}$ for five days).

\section{ELECTROPHYSIOLOGICAL STUDIES}

The patients were tested while reclining and relaxed. Each was asked to keep his or her eyes open and not to fall asleep. The skin temperature of the forearm was maintained above $32^{\circ} \mathrm{C}$. The ulnar nerve was stimulated supramaximally at the wrist, elbow, and axilla using a square current of $0.5 \mathrm{~ms}$ duration, and $M$ waves were recorded from the first dorsal interosseous muscle or abductor digiti minimi muscle by the belly tendon method. Conduction block was diagnosed by the criteria of Unicini et al. ${ }^{8}$ For the $\mathrm{F}$ wave recording, more than 50 consecutive stimuli were delivered to the ulnar nerve at the wrist at a rate of $1 \mathrm{~Hz}$, the intensity being adjusted $20 \%$ higher than that which evoked the maximal $M$ wave. To differentiate $\mathrm{F}$ waves from background noise, only deflections larger than $40 \mu \mathrm{V}$ were accepted. The $\mathrm{F}$ wave frequency was defined as the percentage of stimuli that evoked the $F$ wave. In the six normal controls, the $F$ wave frequency was studied using submaximal stimulation which evoked $M$ waves of $4-16 \%$ of the maximum amplitude.

After giving their informed consent, the patients underwent transcranial magnetic stimulation. A magnetic stimulator (Magstim 200, Magstim Company Ltd, UK) with a high output round coil that had a $9 \mathrm{~cm}$ inner diameter $(2 \cdot 0$ Tesla), was centred over the vertex. Anticlockwise oriented current was used to stimulate the left hemisphere, the adverse side being used to stimulate the right hemisphere. Recordings were made from the first dorsal 
Figure 1 Motor nerve conduction study, $F$ wave and MEP by transcranial magnetic stimulation recorded from the right abductor digiti minimi muscle of patient 10. There was no conduction block in the ulnar nerve between the wrist and Erb and

transcranial magnetic stimulation evoked $M E P$ of $4.0 \mathrm{mV}$ size (left). No $F$ wave was seen (right).

Figure 2 The $F$ wave was absent in patient $8(A)$, but reappeared two days after plasma exchange; the peripheral nerve conduction and the MEP size did not change (B).
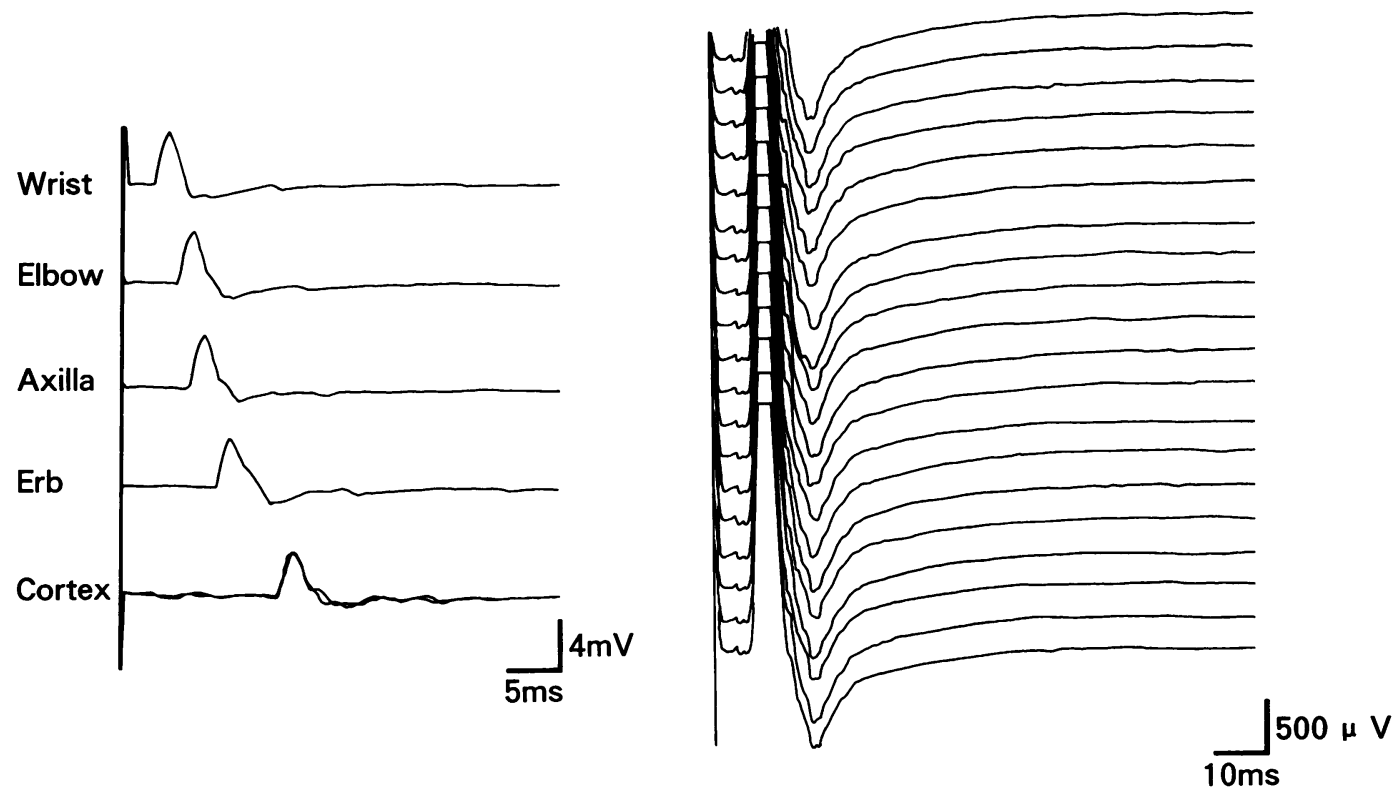

interosseous or abductor digiti minimi muscle. To measure the motor cortical evoked response (MEP), patients were asked to keep the target muscle contracted at $30-50 \%$ of maximum force as monitored by a force transducer. When recordings were made from the first dorsal interosseous muscle, the median nerve was stimulated at the wrist to collide with the volume conducted potential of the

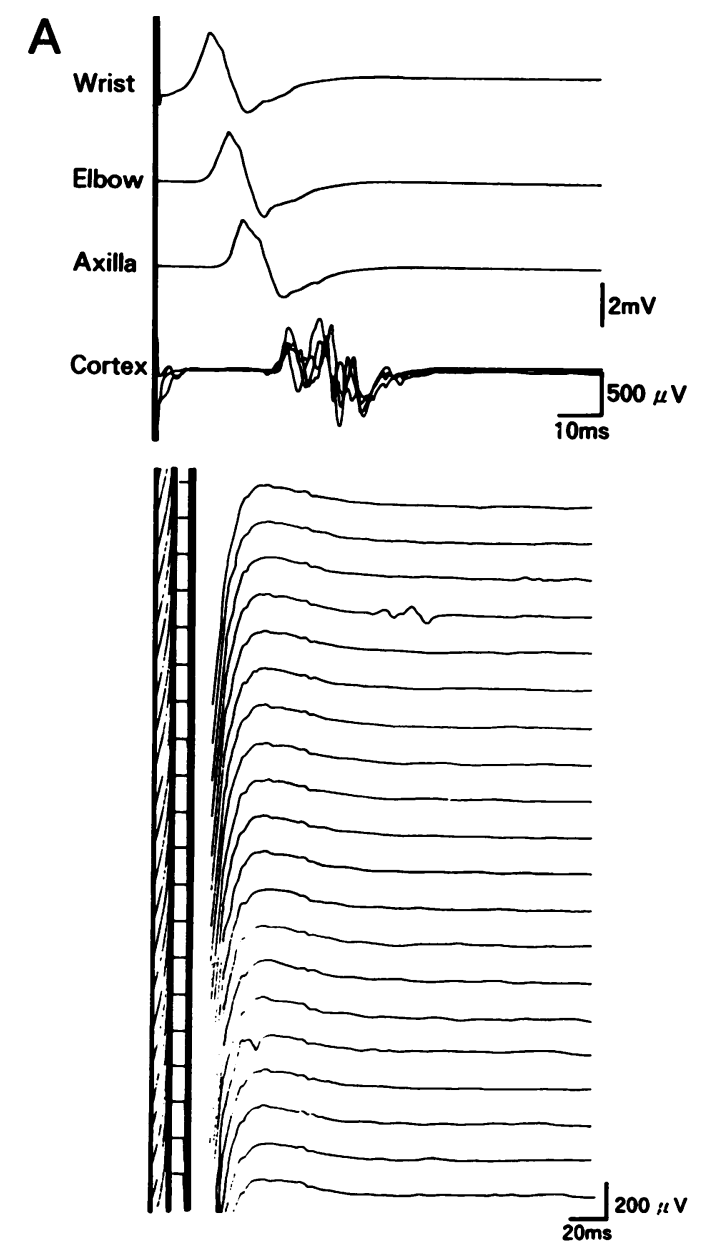

muscles innervated by the median nerve. The stimulus intensity was increased gradually and more than eight cortical stimuli were delivered with the maximum output, from which the largest response was taken as a measure of the peak to peak amplitude.

To determine whether the MEP response was mediated by the same motor neurons producing the $F$ wave, we performed a collision
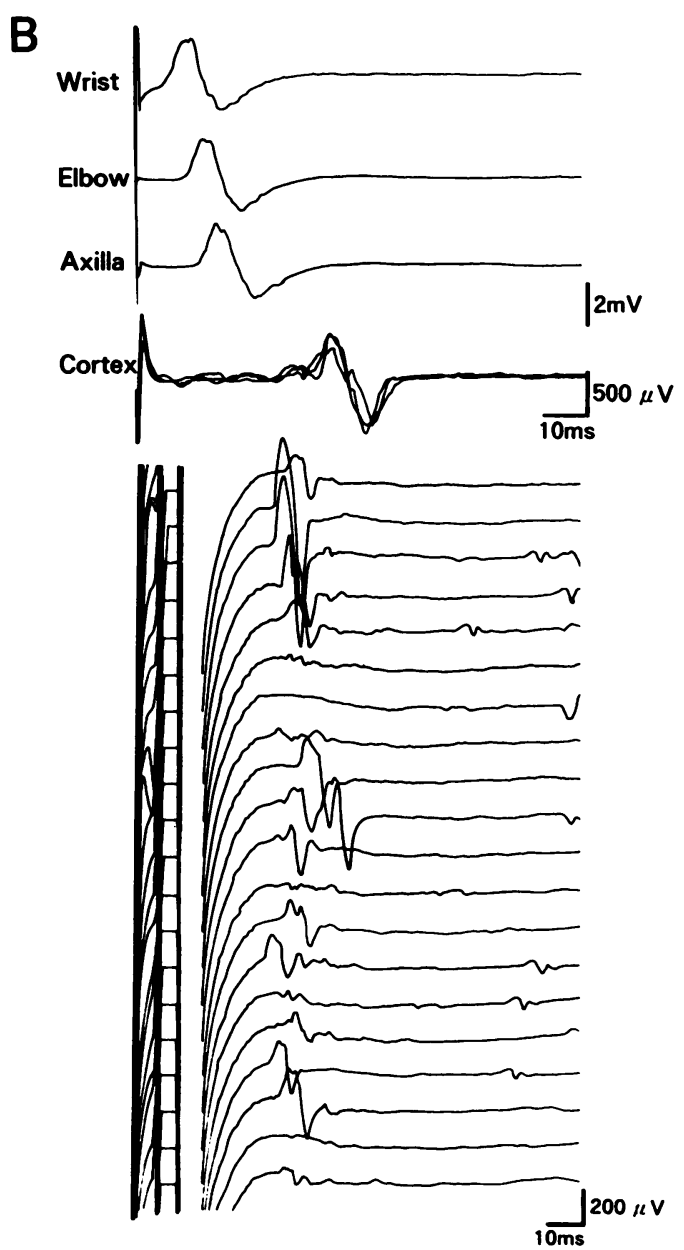
study in three patients with no $\mathrm{F}$ wave but preserved MEPs (patients 5, 7, and 10). For collision of the MEP response, supramaximal triple stimulations were given to the ulnar nerve at the wrist with an interval of $2 \mathrm{~ms}$ at the same time as the transcranial magnetic stimulation, because the later part of the MEP response was evoked by second or third firing of motor neurons produced by transcranial magnetic stimulation. ${ }^{9}$

\section{ANTIGANGLIOSIDE ANTIBODY ASSAYS}

An enzyme linked immunosorbent assay (ELISA) was used to test serum samples from patients 1-21 and from 10 normal subjects for antiganglioside antibodies. Blood samples were taken from patients with Guillain-Barré syndrome within three weeks of the onset of neurological symptoms and from patients with CIDP and MMN in the progressive phase of the illnesses. The ELISA was performed as described elsewhere. ${ }^{10}$ In brief, 10 pmol of each ganglioside were placed in individual slide wells; serum samples (diluted 1:400) were then added. Peroxidase-conjugated goat antihuman $\gamma$ or $\mu$-chain specific antibodies (Tago, Burlingame, CA; diluted to 1:2000) were used as the second antibodies. Each serum sample was tested in triplicate. The absorbance values at $492 \mathrm{~nm}$ were calculated by subtracting values obtained for each well without antigen. Serum was considered positive for a ganglioside if it gave an $\mathrm{OD}_{492}$ of more than 3 SD above the mean value for normal serum.

\section{STATISTICAL ANALYSIS}

Differences in the frequencies of antiganglioside antibody between patients with no $F$ wave and those with a preserved $F$ wave, and between patients with no $F$ wave but preserved MEPs and patients with a preserved $F$ wave were tested for significance by the $\chi^{2}$ test.

\section{Results}

ELECTROPHYSIOLOGICAL STUDIES

The table shows the results of nerve conduction studies and MEP size. Supramaximal amplitude MEPs were obtained in all patients. In the active stage patients 1-10 showed F wave disappearance. Conduction block was noted between the wrist and the axilla in patients 1-6 and 11-16. Patients 1-3 with no $F$ wave had a very attenuated $M$ wave on axilla stimulation (less than $10 \%$ of the normal value) due to conduction block or axonal degeneration; therefore, no MEP was evoked or it was very small $(<0.2 \mathrm{mV})$. The $F$ wave was restored in patient 1 only one day after plasma exchange, even though the MEP size did not change; but, in patients 2 and 3 in whom there was fulminant axonal involvement, their motor nerves were still virtually unexcitable after 10 months of illness. In the other seven patients with no $F$ wave (patients 4-10), however, MEPs of 1-4 mV were recorded (fig 1 ). In patients $4-8$, the $F$ wave reappeared after treatment by plasma exchange or with IVIg, even though the MEP size did not change (fig 2) and there was no

Nerve conduction study, motor evoked potential, and antiganglioside antibodies

\begin{tabular}{|c|c|c|c|c|c|c|}
\hline Patient & $\begin{array}{l}\text { Age/sex } \\
(y)\end{array}$ & Diagnosis & $\begin{array}{l}F \text { wave } \\
\text { frequency }\end{array}$ & $\begin{array}{l}\text { MEP size } \\
(m V)\end{array}$ & $\begin{array}{l}\text { CMAP size } \\
W r / E l / A x(m V)\end{array}$ & $\begin{array}{l}\text { *Antiganglioside } \\
\text { antibody against: }\end{array}$ \\
\hline \multicolumn{7}{|c|}{ Absent $F$ wave with no MEP: } \\
\hline & $24 / \mathrm{M}$ & $\begin{array}{l}\text { GBS } \\
\text { (1 day after PP }\end{array}$ & $\begin{array}{r}0 \\
30\end{array}$ & $\begin{array}{l}0 \cdot 2 \\
0 \cdot 2\end{array}$ & $\begin{array}{l}4 \cdot 8 / 2 \cdot 2 / 0 \cdot 8 \\
4 \cdot 2 / 1 \cdot 6 / 0 \cdot 8)\end{array}$ & $\begin{array}{l}\text { GD1a, GT1b } \\
\text { (IgG, IgM) }\end{array}$ \\
\hline \multirow[t]{2}{*}{2} & $34 / M$ & GBS & 0 & 0 & $0.6 / 0 \cdot 1 / 0 \cdot 1$ & GD1a (IgG) \\
\hline & & (1 month after PP & 0 & 0 & $0 / 0 / 0)$ & \\
\hline 3 & $32 / F$ & CIDP & 0 & 0.2 & $5 \cdot 7 / 3 \cdot 0 / 0 \cdot 9$ & GM1 \\
\hline \multicolumn{7}{|c|}{ Absent $\mathrm{F}$ wave but preserved MEP: } \\
\hline 4 & $31 / \mathrm{F}$ & $\begin{array}{l}\text { MMN } \\
(2 \text { weeks after IVIg }\end{array}$ & $\begin{array}{r}0 \\
40\end{array}$ & $\begin{array}{l}1 \cdot 2 \\
1.4\end{array}$ & $\begin{array}{l}3 \cdot 5 / 1 \cdot 4 / 1 \cdot 3 \\
4 \cdot 6 / 2 \cdot 3 / 2 \cdot 0)\end{array}$ & GM1, GD1b \\
\hline \multirow[t]{2}{*}{5} & $59 / \mathrm{F}$ & GBS & 0 & 1.0 & $8 \cdot 1 / 4 \cdot 2 / 2 \cdot 2$ & GM1, GD1a \\
\hline & & (2 weeks after PP & 75 & 1.0 & $3 \cdot 7 / 2 \cdot 9 / 1 \cdot 1)$ & \\
\hline 6 & $20 / \mathrm{F}$ & $\begin{array}{l}\text { CIDP } \\
(2 \text { weeks after IVIg }\end{array}$ & $\begin{array}{r}0 \\
25\end{array}$ & $\begin{array}{l}1 \cdot 1 \\
1 \cdot 1\end{array}$ & $\begin{array}{l}13 \cdot 6 / 10 \cdot 2 / 3 \cdot 0 \\
17 \cdot 8(16 \cdot 1 / 3 \cdot 3)\end{array}$ & - \\
\hline \multirow[t]{2}{*}{7} & $21 / F$ & GBS & $\begin{array}{r}23 \\
0\end{array}$ & $2 \cdot 4$ & $6 \cdot 7 / 5 \cdot 3 / 5 \cdot 1$ & GM2 \\
\hline & & ( 3 days after PP & 90 & $2 \cdot 1$ & $4 \cdot 2 / 3 \cdot 2 / 2 / 4)$ & \\
\hline \multirow[t]{2}{*}{8} & $76 / F$ & GBS & 0 & 1.0 & $5 \cdot 4 / 5 \cdot 2 / 4 \cdot 1$ & - \\
\hline & & ( 2 days after PP & 65 & $1 \cdot 2$ & $3 \cdot 6 / 3 \cdot 2 / 3 / 2)$ & \\
\hline 9 & 7/M & GBS & 0 & NT & $4 \cdot 6 / 4 \cdot 6$ & GM1, GD1b \\
\hline \multirow{2}{*}{10} & 74/M & $\begin{array}{l}\text { (1 month after IVIg } \\
M M N\end{array}$ & $\begin{array}{l}0 \\
0\end{array}$ & $\begin{array}{l}3 \cdot 0 \\
4 \cdot 0\end{array}$ & $\begin{array}{l}3 \cdot 7 / 3 \cdot 7 / 3 \cdot 7) \\
4 \cdot 3 / 4 \cdot 3 / 4 \cdot 2\end{array}$ & - \\
\hline & $14 / 1012$ & (1 month after IVIg & 0 & $4 \cdot 0$ & $\begin{array}{l}4 \cdot 3 / 4 \cdot 3 / 4 \cdot 2 \\
4 \cdot 3 / 4 \cdot 3 / 4 \cdot 2)\end{array}$ & - \\
\hline \multicolumn{7}{|c|}{ Preserved F wave: } \\
\hline $\begin{array}{l}11 \\
12\end{array}$ & 43/M & GBS & 100 & $1 \cdot 1$ & $7 \cdot 0 / 6 \cdot 3 / 3 \cdot 4$ & - \\
\hline $\begin{array}{l}12 \\
13\end{array}$ & $46 / \mathrm{M}$ & GBS & 78 & $1 \cdot 3$ & $14 \cdot 2 / 8 \cdot 4 / 1 \cdot 9$ & - \\
\hline $\begin{array}{l}13 \\
14\end{array}$ & $72 / \mathrm{M}$ & GBS & 98 & 3.9 & $10 \cdot 9 / 6 \cdot 9 / 5 \cdot 4$ & - \\
\hline $\begin{array}{l}14 \\
15\end{array}$ & $52 / \mathrm{M}$ & CIDP & 100 & 2.9 & $4 \cdot 9 / 2 \cdot 4 / 1 \cdot 3$ & - \\
\hline 15 & $54 / M$ & CIDP & 42 & 0.8 & $3 \cdot 9 / 1 \cdot 4 / 1 \cdot 2$ & NT \\
\hline 16 & $5 / \mathrm{M}$ & GBS & 95 & 0.9 & $7 \cdot 3 / 3 \cdot 3$ & - \\
\hline 17 & $26 / M$ & GBS & 100 & $2 \cdot 5$ & $7 \cdot 0 / 5 \cdot 6 / 5 \cdot 5$ & - \\
\hline 18 & 27/M & GBS & 100 & $3 \cdot 5$ & $9 \cdot 2 / 8 \cdot 2 / 7 \cdot 3$ & - \\
\hline 19 & $59 / \mathrm{F}$ & GBS & 100 & $5 \cdot 3$ & $6 \cdot 5 / 5 \cdot 7 / 4 \cdot 8$ & - \\
\hline 20 & $29 / \mathrm{F}$ & CIDP & 95 & 0.8 & $6 \cdot 1 / 5 \cdot 5 / 3 \cdot 8$ & - \\
\hline 21 & $16 / F$ & CIDP & 80 & $2 \cdot 0$ & $7 \cdot 1 / 6 \cdot 9 / 6 \cdot 6$ & - \\
\hline 22 & $51 / \mathrm{M}$ & CIDP & 100 & $2 \cdot 5$ & $10 \cdot 8 / 6 \cdot 0 / 5 \cdot 8$ & - \\
\hline 23 & $47 / M$ & CIDP & 85 & $4 \cdot 7$ & $16 \cdot 7 / 6 \cdot 9 / 5 \cdot 4$ & - \\
\hline 24 & $24 / M$ & CIDP & 40 & $6 \cdot 1$ & $11 \cdot 8 / 10 \cdot 8 / 10 \cdot 8$ & - \\
\hline 25 & $47 / F$ & CIDP & 90 & $1 \cdot 1$ & $15 \cdot 4 / 13 \cdot 6 / 11.9$ & NT \\
\hline 26 & $62 / \mathrm{M}$ & CIDP & 80 & $1 \cdot 8$ & $9 \cdot 9 / 8 \cdot 5 / 8 \cdot 0$ & NT \\
\hline
\end{tabular}

Italics indicates conduction block

${ }^{\star}$ Isotype of antiganglioside antibodies was only IgM unless otherwise noted.

GBS = Guillain-Barré syndrome; MEP = motor evoked potential by transcranial magnetic stimulation; CMAP = compound muscle action potential; $\mathrm{Wr}_{\mathrm{r}}=$ wrist; $\mathrm{El}=$ elbow; $\mathrm{Ax}=$ axilla; $\mathrm{PP}=$ plasma exchange; $\mathrm{IVIg}=$ intravenous immunoglobulin; $\mathrm{NT}=$ not tested. 
improvement in the target muscle force. By contrast, patients 9 and 10 had no $F$ wave even in the recovery stage.

In the normal controls, when submaximal stimulation produced $M$ waves of $4,8,12$, and $16 \%$ of the maximum amplitude (absolute mean sizes were $1-4 \mathrm{mV}$ ), the mean (range) $F$ wave frequency was $32.5(22-40) \%, 48.0$ $(40-60) \%, \quad 59 \cdot 0(48-88) \%$, and $60 \cdot 0$ (50-90)\% respectively.

The MEPs were collided with almost completely by the peripheral nerve stimulations in all three patients with no $F$ wave but preserved MEPs.

\section{ANTIGANGLIOSIDE ANTIBODIES}

Antibodies to gangliosides GM1, GM2, GD1a, GD1b, or GT1b were positive in seven of the 10 patients with no $F$ wave (table), and the autoantibodies were positive in four of seven patients with no $F$ wave but preserved MEPs. By contrast, the antiganglioside antibodies were negative in the 13 patients with preserved $\mathrm{F}$ waves.

The frequent occurrence of antiganglioside antibodies in the 10 patients with no $F$ wave (patients 1-10) was significant in comparison with the 13 patients with preserved $F$ waves (patients 11-23; $\mathrm{P}<0.01$ ). The frequency of positive antiganglioside antibodies in seven patients with no $F$ waves but preserved MEPs (patients 4-10) was higher than those of the patients with preserved $F$ waves $(P<0.05)$.

\section{Discussion}

In seven of the 10 patients with no $F$ waves, transcranial magnetic stimulation on the motor cortex elicited MEPs of 1-4 mV. The MEPs did not include volume conducted potentials of activated muscles other than the target muscle, because MEPs were collided by the stimulation of the peripheral nerve innervating the target muscle. These indicated that there was preserved conduction through all the segments of the peripheral motor nerve fibres on which stimulation evoked an $M$ wave larger than the MEP in amplitude. Because the multiple descending volleys produced by cortical shock cause asynchronous activation of motor units, and the EMG responses from each unit interfere, this reduces the maximal amplitude of the response and increases its duration. Cortical stimulation may activate the motor unit more than once ${ }^{9}$ but a second firing of the unit may contribute to the later part of the compound EMG response, and have little influence on the amplitude of its main component.

In normal subjects, even with submaximal peripheral nerve stimulation that evoked a small $M$ wave of $1 \mathrm{mV}$ which was $4 \%$ of the maximum value, an $\mathrm{F}$ wave of more than $22 \%$ frequency could be recorded. However, proximal conduction abnormality may still explain the absence of $F$ waves with preserved MEPs if the number of motor neurons that will be excited with transcranial magnetic stimulation are considerably greater than those which will be excited by the $F$ wave pulse. However, a recent study of motor unit number estimates using $\mathrm{F}$ waves indicates that almost all motor neurons contribute to the $\mathrm{F}$ wave. ${ }^{11}$ Moreover, the $F$ waves reappeared in the patients after treatment, even when there was no or little improvement in peripheral nerve conduction between the wrist and axilla and MEP size. Therefore, in the patients with no $F$ waves but preserved MEPs, the MEP response was mediated by the same motor neurons that could produce the F wave. Such severe conduction block with no MEPs as seen in patients $1-3$ is an important factor for $F$ wave disappearance. However, our results suggest that the popular pathophysiology of $F$ wave disappearance in demyelinating neuropathies is not conduction block at the proximal site, but impairment of motor neuron excitability, or prolonged refractoriness of the most proximal axon for backfiring in the patients with preserved MEPs.

The rapid reappearance of the $F$ wave after treatment by plasma exchange or with IVIg indicates that such circulating factors as autoantibodies to nerve tissue function by blocking the generation of the $\mathrm{F}$ wave. The pathological relevance of antiganglioside antibodies has not been established in vivo; but autoantibodies affect the nodal and motor neuron excitability in vitro. Sodium current at the nodes is decreased by anti-GM1 antibody in the presence of complement in isolated single nerve fibres from rats. ${ }^{12}$ Monoclonal antibodies to GM1 and GM2 reversibly suppressed miniature endplate potentials and spontaneous firings in a heterotypic coculture system of human muscle cells and fetal rat spinal cord explants. ${ }^{13}$ Spinal motor neurons have $\mathrm{GM} 1^{14}$ or the $\mathrm{Gal}(\beta 1-3)$-GalNAc epitope that is shared by GM1 and GD1b. ${ }^{15}$ Therefore, antiganglioside antibodies are candidates for blockers of $F$ wave generation. Our results support this hypothesis, but conclusive evidence requires a study of more patients. To validate the hypothesis, we will next investigate in vitro whether antiganglioside antibodies block $\mathrm{F}$ wave generation.

1 Kimura J. F wave. In: Electrodiagnosis in diseases of nerve and muscle: principle and practice. 2nd ed. Philadelphia: FA muscle: principle and

2 Ad hoc subcommittee of the American Academy of Neurology AIDS Task Force. Research criteria for diag nosis of chronic inflammatory demyelinating polyradicuoneuropathy. Neurology 1991;41:617-8.

3 Fisher MA, Shahani BT, Young RR. Assessing segmental excitability after acute rostral lesions. Neurology 1978;22 1265-71.

4 Yokota T, Shimizu T, Hayashi H, et al. F-responses during cataplexy. I Neurol Neurosurg Psychiatry 1992;55:75-6.

5 Fisher MA, Penn RD. Evidence for changes in segmenta motoneurone pools by chronic cerebellar stimulation and ts clinical significance. $f$ Neurol Neurosurg Psychiatry 1978;41:630-5.

6 Asbury AK, Cornblath DR. Assessment of current diagnostic criteria for Guillain-Barré syndrome. Ann Neurol tic criteria for

7 Chaudhry V, Corse AM, Cornblash DR, et al. Multifocal motor neuropathy: response to human immune globulin. Ann Neurol 1993;33:237-42.

8 Uncini A, Di Muzio A, Sabatelli M, et al. Sensitivity and specificity of diagnostic criteria for conduction block in chronic inflammatory demyelinating polyneuropathy. Electroencephalogr Clin Neurophysiol 1993;89:161-9.

9 Day BL, Rothwell JC, Thompson PD, et al. Motor cortex stimulation in intact man. 2. Multiple descending volleys. Brain 1987;110:1191-209.

10 Yuki N, Yoshino H, Sato S, Shinozawa K, Miyatake T. Severe acute axonal form of Guillain-Barré syndrome associated with IgG anti-GDla antibodies. Muscle Nerve 1992;15:899-903. 
11 Stashuk DW, Doherty TJ, Kassam A, Brown WF. Motor unit number estimates based on the automated analysis of F-responses. Muscle Nerve 1994;17:881-90.

12 Takikawa $T$, Yasuda $\mathrm{H}$, Kikkawa $\mathrm{R}$, et al. Antibodies against $\mathrm{GM}_{1}$ ganglioside affect $\mathrm{K}^{+}$and $\mathrm{Na}^{+}$current in isolated rat myelinated nerve fibres. Ann Neurol 1995;37: 436-42.

13 Kobayashi T, Michikawa M, Park-Mastumoto YC, et al. Inhibition of motor neuron excitability by serum with
anti-GM1 and GM2 antibodies and their monoclonal ones. Can $\mathcal{F}$ Neurol Sci 1993;20(suppl):S134

14 Corbo M, Quattrini A, Lugaresi A, et al. Patterns of reactivity of human anti-GM1 antibodies with spinal cord tivity of human anti-GM antibodies with spinal

15 Lugaresi A, Corbo M, Thomas FP, et al. Identification of glycoconjugates which are targets for anti-Gal $(\beta 1-3)$ GalNAc autoantibodies in spinal motor neurons. $\mathcal{F}$ Neuroimmunol 1991;34:69-76. 